\title{
Vasera-screening of premature vascular aging and its correction in young persons: University Health Center experience
}

\author{
Maria Evsevyeva $^{1,{ }^{*}}$, Mikhail Eremin ${ }^{2}$, Elena Fursova $^{1}$, Anjelika Rusidi $^{1}$, and Evgenij \\ Shchetinin ${ }^{1}$ \\ ${ }^{1}$ Stavropol State Medical University, Internal Diseases Department, 355017, Mira St., 310, Stavropol, \\ Russia \\ ${ }^{2}$ Stavropol Regional Clinical Hospital, Otorhinolaryngology Department, 355030, Semashko St, 1, \\ Stavropol, Russia
}

\begin{abstract}
Angiological screening was performed to determine the cardio-ankle vascular index (CAVI) in 224 young people to determine their vascular aging phenotype. All subjects were divided into CAVI-tercile groups. According to the experts views the upper tercile of this distribution corresponds to early vascular aging or EVA syndrome. Lower tercile corresponds to favorable one and average tercile - to normal vascular aging. In these CAVI-tercile groups, the representation of individuals with signs of arterial hypertension/prehypertension (AH/PH) and overweight was evaluated. It turned out that among boys and girls with risk factors (RF), carriers of various vascular phenotypes are noted, from favorable one to early vascular aging. Such data indicate the need to form youth risk groups, taking into account the presence of not only classical RF, but also the assessment of vascular stiffness. The obtained data is useful to take into account in the process of youth medical examination in order to further develop more individualized preventive intervention programs for each young person.
\end{abstract}

\section{Background}

Worldwide, cardiovascular (CV) mortality remains at a high level [1], due to a wide spread of risk factors (RF), including premature vascular aging, determined by the level of arterial wall stiffness, which is regarded now as implemented RF [2]. It is proved that this marker of $\mathrm{CV}$ risk has a higher prognostic potential than traditional RF [3]. From a biological point of view, it is the vessels that begin to age earlier than other organs and tissues. For this reason, it is necessary to establish a system of wide angiological screening among young people as soon as possible, since this group has the maximum chance of inhibiting vascular remodeling in the case of preventive intervention [4]. At the same time, it is desirable to

* Corresponding author: evsevieva@mail.ru 
evaluate the identified RF in relation to the belonging of the subject to a particular vascular aging phenotype. The features of such associations are clearly insufficiently studied, as well as the possibility of using the vascular age indicator to motivate young people to conduct preventive interventions and monitor their effectiveness

Aim - to determine the features of associations of various vascular aging phenotypes with certain RF in young people from the standpoint of tercile analysis of the arterial stiffness index, as well as to study its dynamics in the process of correcting of behavioral $\mathrm{RF}$ in the framework of an intra-University preventive project.

\section{Material and Methods}

Angiological screening was performed for 224 young people (81 boys and 162 girls) aged 18-25 years (group 1) on the basis of the StSMU Health Center as part of the National Preventive Project "University Territory of Health". Another 382 students (2nd group) was performed only antropometria and questionnaires. Angiological screening was performed using the VASERA VS-1500 device ('Fukuda Denshi', Japan), which allows us to evaluate such an indicator of arterial stiffness as the cardio-ankle vascular index (CAVI) [5,6]. This indicator, unlike others, does not depend on the level of stretching blood pressure acting on the arterial wall at the time of registration of the pulse wave. The method is based on recording phonocardiography, determining the heart tone II, plethysmography, which registers pulse waves on the shoulder and popliteal artery from both sides through the cuff, and then transforms the function. All sex-adjusted subjects were divided into tercile groups based on the CAVI and gender. According to the views of leading experts [4], the upper tercile of this distribution corresponds to premature vascular aging or EVA-syndrome (early vascular aging). Lower tercile corresponds to favorable one and average - to normal vascular aging. In these CAVI-tercile groups, the representation of individuals with signs of arterial hypertension/prehypertension (AH/PH) and overweight was evaluated. The obtained data were processed using the statistical software package "Statistica 10.0" (StatSoft Inc,USA).

\section{Results and Discussion.}

According to the data of the tercile analysis, carriers of EVA-syndrome were characterized by the presence of CAVI from 6.1 to 8.1, and carriers of favorable vascular aging differed in CAVI from 3.2 to 5.6. Distribution of students with $\mathrm{AH} / \mathrm{PH}$ from the lower to upper tercile group for young men was as follows: $21.0 \%, 18.5 \%$ and $12.3 \%$ of all the examined boys. For girls, this ratio was $2.5 \%, 4.9 \%$ and $2.5 \%$ of the total number of girls surveyed. Among boys the incidence of overweight in these groups varied as follows: $22.2 \%, 8.6 \%$ and $6.2 \%$ of all boys, and among girls $-4.4 \%, 2.5 \%$ and $2.5 \%$ of all girls. Among the 1st group students, $87.8 \%$ expressed a desire to participate in the prevention program, and $65.4 \%$ completed educational training. In the second group,there were only $28.6 \%(\mathrm{P}<0,05)$ and $22.6 \%(\mathrm{P}<0,05)$. This correction of behavioral stereotypes in students was carried out in the conditions of the University School of Student Health, organized on the basis of the Health Center with the inclusion of a psychologist and psychotherapist in the team. To date, during the control angiological study in seven people who followed the recommendations for RF correction for six months, a decrease in the CAVI-level was detected by an average of $9.5 \pm 1.1 \%$. O ur data are consistent with the opinion of other authors on the feasibility of early predictive diagnostics of vascular phenotype at a young age [5]. The instrumental angiological screening we used among young people is optimally suited for this purpose. These results show that it is no longer possible to limit the use of classical 
RF [7,8], as was done until recently when conducting youth screening. Predictive diagnostic advantages of vascular stiffness in comparison with other RF are due to its nature as a "tissue" biomarker in comparison with "circulating" biomarkers such as lipids, C-reactive protein, homocysteine, etc., the level of which constantly fluctuates in the human body [9]. It is believed that arterial stiffness integrates the long-term effect of all present RF [2]. Timely recognition of cases with increased arterial rigidity helps to exclude the erroneous inclusion of a such patients in a low-risk group. The use of this method in the course of medical examination will contribute to the implementation of timely, and therefore effective predictive diagnostics at a young age. Early verification of the vascular phenotype will allow optimizing preventive interventions towards their maximum individualization, taking into account the peculiarities of the vascular status of each young person [10]. An argument in favor of the introduction of angiological technologies in the field of youth preventology is the evidence that the vascular wall is involved in the process of biological aging earlier than other organs and systems [11-12]. In the case of further clinical manifestations of a particular atherosclerotic CV disease, the presence of a vascular history with data on the dynamics of the vascular phenotype will contribute to a more personalized treatment of the detected pathology [13]. Therefore, it is necessary to improve the system of mass diagnostic measures within the framework of organized youth groups [14], in order to further conduct individualized preventive measures of anti-aging orientation.

\section{Conclusion}

Thus, both among boys and girls with the presence of such traditional RF as AH/PH and overweight the carriers of various vascular phenotypes from favorable to early vascular aging are noted. Such data indicate the need to form youth groups of CV risk, taking into account the presence of not only classical RF, but also the assessment of vascular stiffness, which allows us to correlate each young person with a certain phenotype of vascular aging. The vascular stiffness index is also useful for monitoring the effectiveness of preventive measures.

\section{References}

1. S. Boitsov, A. Demkina, E. Oshchepkova, Yu. Dolgusheva, Cardiology. 59, 3 (2019) doi.org/10.18087/cardio.2019.3.10242

2. P.M. Nilsson J Hypertens., 31,8 (2013)

3. Y. Ben-Shlomo, M. Spears, C. Boustred, et al. J Am Coll Cardiol. 63, 636-646 (2014). doi: 10.1016/j.jacc.2013.09.063

4. S. Laurent, P. Boutouyrie, P. Cunha, P. Lacolley, P. Nilsson, Hypertension, 74, 218 228 (2019)

5. A. Takaki, H. Ogawa, T. Wakeyama, et al. Hypertens Res., 31, 7 (2008) doi: 10.1291/hypres.31.1347

6. A. Sumin, A. Osokina, A. Shcheglova, et al., Heart. 14, 3 (2015)

7. M. Evsevyeva, E. Miridzhanjan, I. Babunts, Yu. Pervushin, Cardiovascular therapy and prevention, 4, 6-2 (2005)

8. M. Evsevyeva, Z. Kumukova, Rus Psychiatr J., 3, 53-57 (2007)

9. I. Strazhesko, O. Tkacheva, D. Akasheva, et al., Rational Pharmacotherapy in Cardiology, 12, 3 (2016) doi.org/10.20996/1819-6446-2016-12-3-244-252

10. S. Gielen, G. Backer, M. Piepol, et al., The ESC Textbook of Preventive Cardiology (Oxford, University Press, 2016) 
11. N. Kononova, T. Chernyshova, R. Zagrtdinova, Arch int med.; 7(4) 287 - 291 (2017) DOI: 10.20514/2226-6704-2017-7-4-287-291

12. N. Kutakova, T. Shushkova, T. Yudina, Bull Rus State Med Univ., 12, $32-38$ (2013)

13. O. Golubnitschaja, V. Costigliola, EPMA J., 3, 1 (2012) doi:10.1186/1878-5085-3-14

14. M. Evsevyeva, V.Koshel, M. Eremin, I. Gal'kova, A. Rusydi, E. Chudnovsky, et al., Med Bull North Caucasus., 10, 1 (2015) doi:10.14300/mnnc.2015.10009 